\title{
Bounded-to-1 factors of an aperiodic shift of finite type are 1-to-1 almost everywhere factors also
}

\author{
JONATHAN ASHLEY \\ Department of Mathematical Sciences, IBM Thomas $J$ Watson Research Center, \\ PO Box 218, Yorktown Heights, NY 10598, USA
}

(Recelved 18 October 1988)

\begin{abstract}
We show that if $\pi \Sigma_{G} \rightarrow \Sigma_{H}$ is a bounded-to-1 factor map from an irreducible shift of finite type $\Sigma_{G}$ with period $p_{G}$ to a shift of finite type $\Sigma_{H}$ with period $p_{H}$, then there is a factor map $\hat{\pi} \Sigma_{G} \rightarrow \Sigma_{H}$ that is $\left(p_{G} / p_{H}\right)$-to-1 almost everywhere Moreover, if $\pi$ is right closing, then $\hat{\pi}$ may be taken to be right closing also
\end{abstract}

\section{Introduction}

We prove the following result

THEOREM 11 If $\pi \quad \Sigma_{G} \rightarrow \Sigma_{H}$ is a bounded-to-1 factor map from an irreductble shift of finte type $\Sigma_{G}$ with period $p_{G}$ to a shift of finite type $\Sigma_{H}$ with period $p_{H}$, then there is a factor map $\hat{\pi} \quad \Sigma_{G} \rightarrow \Sigma_{H}$ that is $\left(p_{G} / p_{H}\right)$-to-1 almost everywhere Moreover, if $\pi$ is right closing, then $\hat{\pi}$ may be taken to be right closing also

In particular, if $\Sigma_{G}$ is aperiodic, then $\hat{\pi} \quad \Sigma_{G} \rightarrow \Sigma_{H}$ is 1-to-1 almost everywhere It is easy to show that $p_{G} / p_{H}$ is the smallest possible degree of a factor map from a shift of period $p_{G}$ to a shift of period $p_{H}$

This result generalizes a result in [AGW] where the range shift is the full $n$-shift

As was pointed out to me by Bruce Kitchens and Brian Marcus, this result simplifies the proof of the main theorem in [AM] that topological entropy and period are a complete set of invariants for almost topological conjugacy

\section{Background}

We assume some familiarity with shifts of finite type $\S 3$ of [AM] and $\S 2$ of [BMT] are good introductions We make some definitions here in order to establish notation

Given a strongly connected directed graph $G$ with a finite set of states $\mathscr{S}$ and at most one edge from any state to any other, we define the shift of finite type $\Sigma_{G}$ by

$$
\Sigma_{G}=\left\{s \in \mathscr{G}^{\mathbb{Z}} \quad s_{t} s_{t+1} \text { is an edge in } G \text { for } I \in \mathbb{Z}\right\}
$$

This definition follows [AM] rather than [BMT] In [BMT] the defining graph $G$ may have many parallel edges from one state to another, and the symbols in the shift $\Sigma_{G}$ are the edges of $G$, not the states of $G$ The definitions are equivalent up to conjugacy 
The set $\Sigma_{G}$ is topologized by the product of the discrete topologies on its coordinate spaces

The shift map $\sigma \quad \Sigma_{G} \rightarrow \Sigma_{G}$ defined by

$$
(\sigma x)_{t}=x_{i+1}
$$

is a homeomorphism

The period of $\Sigma_{G}$ is the greatest common divisor of all cycle lengths in the graph $G$ Given a finite path of states $s_{1} s_{2} \quad s_{k}$ in the graph $G$, we denote

$$
{ }_{n}\left[s_{1} s_{2} \quad s_{k}\right]_{n+k-1}=\left\{x \in \Sigma_{G} \quad x_{n+1-1}=s_{1}, 1 \leq \imath \leq k\right\}
$$

This set called a $k$-block of $\Sigma_{G}$

Given $y \in \Sigma_{G}$, we denote the finite path $y_{i} y_{i+1} \quad y_{J}$ in $G$ by ${ }_{1}(y)$,

A $k$-block map $\pi \Sigma_{G} \rightarrow \Sigma_{H}$ is a shift-commuting map such that there is some $l$ for which

$$
(\pi y)_{0}=\left(\pi y^{\prime}\right)_{0} \text { if } t_{t-k+1}(y)_{l}={ }_{t-k+1}\left(y^{\prime}\right)_{l}
$$

In the 1-block case we require merely for notational convenience that $l=0$ In the 1-block case we have

$$
(\pi y)_{0}=\left(\pi y^{\prime}\right)_{0} \quad \text { if } y_{0}=y_{0}^{\prime}
$$

Thus $\pi$ is defined by a map from single states of $G$ to single states of $H$ that we again call $\pi$ In this case we say that a path of states $s_{1} s_{2} \quad s_{k}$ in $G$ is $\pi$-labelled by $\pi\left(s_{1}\right) \pi\left(s_{2}\right) \quad \pi\left(s_{k}\right)=\pi\left(s_{1} s_{2} \quad s_{k}\right)$

A bounded-to-1 factor map $\pi \Sigma_{G} \rightarrow \Sigma_{H}$ is a $k$-block map such that the set of positive integers $\left\{\# \pi^{-1}(y) \quad y \in \Sigma_{H}\right\}$ is bounded from above

A 1-block map $\pi \Sigma_{G} \rightarrow \Sigma_{H}$ is right-closing if it never identifies two distinct left asymptotic points if $s, s^{\prime} \in \Sigma_{G}$ have an $l_{0} \in \mathbb{Z}$ such that $s_{l}=s_{l}^{\prime}$ for all $l \leq l_{0}$ and $\pi(s)=\pi\left(s^{\prime}\right)$ then $s=s^{\prime}$

A 1-block map $\pi \quad \Sigma_{G} \rightarrow \Sigma_{H}$ is right-resolving if for every path $t_{1} t_{2}$ of length 2 in $H$, and for every state $s_{1}$ of $G$ with $\pi\left(s_{1}\right)=t_{1}$, there is a unique state $s_{2}$ such that $s_{1} s_{2}$ is an edge of $G$ and $\pi\left(s_{2}\right)=t_{2}$

\section{Resolving blocks}

If $\pi \Sigma_{G} \rightarrow \Sigma_{H}$ is bounded-to-1, then the minimum $d$ of $\left\{\# \pi^{-1}(y) y \in H\right\}$ is the genenc degree of $\pi$ except for a set of measure zero in $\Sigma_{G}$ (with respect to the measure of maximal entropy) $\pi$ is a $d$-to-1 map [KMT] We call $d$ the degree of $\pi$ after [B]

The degree of a 1-block factor map $\pi \Sigma_{G} \rightarrow \Sigma_{H}$ is the smallest integer $d$ such that there is a path $m_{1} m_{2} \quad m_{k}$ in the graph $H$, an integer $l, 1 \leq l \leq k$, and a set $\left\{r^{1}, r^{2}, \quad, r^{d}\right\}$ of $d$ states in the graph $G$ such that every path $s_{1} s_{2} \quad s_{k}$ in $G$ with $\pi\left(s_{1} s_{2} \quad \cdot s_{k}\right)=m_{1} m_{2} \cdots m_{k}$ has $s_{l} \in\left\{r^{1}, r^{2}, \quad, r^{d}\right\}$ [KMT] The path $m_{1} m_{2} \quad m_{k}$ is a resolving block for the map $\pi$ We use the following construction from [KMT] to reduce to a convenient special case of Theorem 11

Given a shift of finite type $\Sigma_{H}$ define the $k$-block presentation of $\Sigma_{H}$ to be the shift of finite type $\Sigma_{H}^{[k]}$ whose symbols are the paths of length $k$ in $H$, with a transition from symbol $s_{1} s_{2} \quad s_{k}$ to symbol $t_{1} t_{2} \quad t_{k}$ iff $s_{2} s_{3} \quad s_{k}=t_{1} t_{2} \quad t_{k-1}$ The $k$-block 
map $\psi_{k} \Sigma_{H} \rightarrow \Sigma_{H}^{[k]}$ defined by mapping the path $s_{1} s_{2} \quad s_{k}$ in $H$ to the symbol $s_{1} s_{2} \quad s_{k}$ in $\Sigma_{H}^{[k]}$ is a conjugacy

Given a 1-block map $\pi \Sigma_{G} \rightarrow \Sigma_{H}$ and integers $k$ and $l$ with $1 \leq l \leq k$, define the shift of finite type $\Sigma_{G}^{k, l}$ as follows The symbols to $\Sigma_{G}^{k l}$ are the equivalence classes of paths of length $k$ in $G$ where path $s_{1} s_{2} \quad s_{k}$ is equivalent to $s_{1}^{\prime} s_{2}^{\prime} \quad s_{k}^{\prime}$ iff

$$
\pi\left(s_{1} s_{2} \quad s_{k}\right)=\pi\left(s_{1}^{\prime} s_{2}^{\prime} \quad s_{k}^{\prime}\right)
$$

and

$$
s_{l}=s_{l}^{\prime}
$$

There is a transition in $\Sigma_{G}^{k l}$ from equivalence class $s$ to equivalence class $t$ iff there is a path $s_{1} s_{2} \quad s_{k} s_{k+1}$ in $G$ such that $s_{1} s_{2} \quad s_{k} \in s$ and $s_{2} s_{3} \quad s_{k+1} \in t$ The $k$-block map $\varphi_{k, l} \Sigma_{G} \rightarrow \Sigma_{G}^{k l}$ taking a path of length $k$ in $G$ to the equivalence class containıng it is a conjugacy

Define the 1-block map $\pi_{k, l} \Sigma_{G}^{k, l} \rightarrow \Sigma_{H}^{[k]}$ by takıng a symbol of $\Sigma_{G}^{k, l}$ (which is an equivalence class of paths of length $k$ in $G$ ) to the common $\pi$-label of its elements

ThEOREM 31 ([KMT]) The diagram

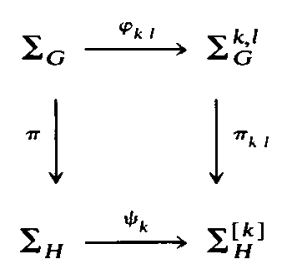

commutes Moreover, if $m_{1} m_{2} \quad m_{k}$ is a path in $H$ that is a resolving block for $\pi$, and $l, 1 \leq l \leq k$, is as in the definition of a resolving block, then $m_{1} m_{2} \quad m_{k}$ is a resolving symbol for $\pi_{k, l}$

We also use the following lemma essentially contained in [KMT] regarding bounded-to-1 factor maps

Permutation LEMma 32 Let $\pi \Sigma_{G} \rightarrow \Sigma_{H}$ be a degree d 1-block map with resolving symbol $m$ Let $m^{1}, m^{2}, \quad, m^{d}$ be the states in $G$ with $\pi\left(m^{\prime}\right)=m, 1 \leq 1 \leq d$ For each path of the form mum in $H$, there are paths $u^{1}, u^{2}, \quad, u^{d}$ in $G$ and a permutation $\tau_{u}$ of $\{1,2, \quad, d\}$ such that the paths of $G \pi$-labelled by mum are exactly $m^{\prime} u^{\prime} m^{\tau_{u}(1)}$, $1 \leq 1 \leq d$

\section{Proof of the main theorem}

In case the entropy of $\Sigma_{G}$ is zero, $\Sigma_{G}$ and $\Sigma_{H}$ each consist of a single finite orbit and Theorem 11 holds trivially The rest of this section treats the positive entropy case

We first reduce to a special case Suppose the given map $\pi \Sigma_{G} \rightarrow \Sigma_{H}$ is a $k$-block map

As composition (on either side) with conjugacies preserves both degree and the property of being right-closing, we can reduce to the case where $\pi \Sigma_{G} \rightarrow \Sigma_{H}$ is a 
1-block map by replacing $\pi$ with $\varphi \circ \pi$ where

$$
\varphi \quad \Sigma_{G}^{[k]} \rightarrow \Sigma_{G}
$$

1s the 1-block conjugacy mapping the word $s_{1} s_{2}$

$s_{k}$ in $\Sigma_{G}$ to the symbol $s_{k}$

Using Theorem 31 , we further reduce to the case that $\pi \Sigma_{G} \rightarrow \Sigma_{H}$ is a 1-block map with a resolving symbol $m$ We may assume, by increasing $k$ in Theorem 31 if necessary, that the resolving symbol $m$ in $H$ has at least two incoming edges and at least two outgoing edges in $H$ The motive here will not become apparent until later

Assuming $\pi \Sigma_{G} \rightarrow \Sigma_{H}$ has degree exceeding $p_{G} / p_{H}$, we will construct a boundedto-1 factor map $\hat{\pi} \Sigma_{G} \rightarrow \Sigma_{H}$ that has lower degree than $\pi$ Moreover $\hat{\pi}$ will be right-closing if $\pi$ is Since any factor map from $\Sigma_{G}$ to $\Sigma_{H}$ has degree at least $p_{G} / p_{H}$, this will prove that there is a factor map from $\Sigma_{G}$ to $\Sigma_{H}$ with degree exactly $p_{G} / p_{H}$

First we construct $\hat{\pi}$ and then show that it has the desired properties

If the graph $G$ has period $p_{G}$, then the states of $G$ are partitioned into $p_{G}$ equivalence classes $\mathscr{C}_{0}, \mathscr{C}_{1}, \quad, \mathscr{C}_{p_{G}-1}$, where a state $s$ is equivalent to a state $t$ iff there is a path sut in $G$ with $|u t|$ a multiple of $p_{G}$

Let $m^{1}, m^{2}, \quad, m^{d}$ be the symbols in $G$ with $\pi\left(m^{l}\right)=m, 1 \leq \imath \leq d$ Since $\Sigma_{H}$ has period $p_{H}$, any cycle based at $m$ has length a multiple of $p_{H}$ So we may assume that all the symbols $m^{1}, m^{2}, \quad, m^{d}$ occur in the equivalence classes

$$
\mathscr{C}_{0}, \mathscr{C}_{p_{H}}, \mathscr{C}_{2 p_{H}}, \quad, \mathscr{C}_{\left(p_{G} / p_{H}-1\right) p_{H}}
$$

Thus $d$ objects are placed in $p_{G} / p_{H}$ pigeon holes If we assume $d>p_{G} / p_{H}$, then two of $m^{1}, m^{2}, \quad, m^{d}$ lie in the same equivalence class We may assume these two are $m^{1}$ and $m^{2}$ and that $m^{1}, m^{2} \in \mathscr{C}_{0}$

Fix $N_{0}>0$ such that for any $0 \leq 1, J<p_{G}$, any state $s$ in $\mathscr{C}_{l}$ and any state $t$ in $\mathscr{C}_{J}$, there is a path of length $(J-l)+N_{0} p_{G}$ from state $s$ to state $t$

Let $e_{2} e_{3} \quad e_{L}$ be a (possible empty) path in $H$ such that $m e_{2} e_{3} \quad e_{L}$ is a simple cycle in $H$ Denote $m=e_{1}$ Recall that $m$ has at least two incoming and at least two outgoing edges Choose states $f$ and $h$ in $H$ so that $m f$ and $h m$ are edges of $H$ not occurring on the cycle $e_{1} e_{2} \quad e_{L}$

Choose an integer $p$ such that $p L+1 \geq p_{G}+N_{0} p_{G}+1$

By the Permutation Lemma 32 there is a path $c_{1} c_{2} \quad c_{p L+1} s_{0}=c s_{0}$ in $G$ with $c_{1}=m^{1}$ and with $\pi$-label $\left(e_{1} e_{2} \quad e_{L}\right)^{p} e_{1} f$ Again by the permutation Lemma 32 , there is a state $s_{N}$ of $G$ such that $s_{N} m^{1}$ is an edge of $G$ and $\pi\left(s_{N}\right)=h$ Similarly, there is a state $\bar{s}_{N}$ of $G$ such that $\bar{s}_{N} m^{2}$ is an edge of $G$ and $\pi\left(\bar{s}_{N}\right)=h$

Now $s_{0} \in \mathscr{C}_{p L+1}$ and $s_{N}, \bar{s}_{N} \in \mathscr{C}_{-1}$ (indices are $\bmod p_{G}$ ) Fix $I_{0}$ with $I_{0} \equiv-1-(p L+1) \bmod p_{G}$ and $0 \leq I_{0}<p_{G}$ Set $N=I_{0}+N_{0} p_{G}$ We may choose a path $s_{0} s_{1} \cdot s_{N-1} s_{N}$ from state $s_{0}$ to state $s_{N}$ and a path $s_{0} \bar{s}_{1} \quad \bar{s}_{N-1} \bar{s}_{N}$ from state $s_{0}$ to state $\bar{s}_{N}$ Denote $s_{0}=\bar{s}_{0}$ By the choice of $p$, we have

$$
p L+1 \geq p_{G}+N_{0} p_{G}+1 \geq I_{0}+N_{0} p_{G}+2=N+2,
$$

an inequality we will use in the proof of Lemma 41 below

Denote $M=p L+1+N+2$ and

$$
t=t_{1} t_{2} \quad t_{M}=\left(\begin{array}{lll}
c_{1} c_{2} & \left.c_{p L+1}\right)\left(s_{0} s_{1}\right. & \left.s_{N}\right) m^{1}
\end{array}\right.
$$


and

$$
\bar{t}=\bar{t}_{1} \bar{t}_{2} \quad \bar{t}_{M}=\left(\begin{array}{lll}
c_{1} c_{2} & c_{p L+1}
\end{array}\right)\left(\bar{s}_{0} \bar{s}_{1} \quad \bar{s}_{N}\right) m^{2}
$$

Denote $\pi\left(s_{1} s_{2} \quad s_{N-1}\right)=g, \pi\left(\bar{s}_{1} \bar{s}_{2} \quad \bar{s}_{N-1}\right)=\bar{g}$, and $\left(e_{1} e_{2} \quad e_{L}\right)^{p} e_{1}=e$ Note that $\pi(t)=e f g h m$ and $\pi(\bar{t})=e f \bar{g} h m$ Thus $g \neq \bar{g}$ by Lemma 32

The paths $t$ and $\bar{t}$ in $G$ were chosen in part to make the following lemma true

LEMMA 41 The two paths $\pi(t)=m v m=e f g h m$ and $\pi(\bar{t})=m \bar{v} m=e f \bar{g} h m$ in H nontrivially overlap each other or themselves only at their end symbols, $m$

Proof Say path $u$ encroaches upon path $w$ by $n$ if $u=u^{\prime} s, w=s w^{\prime}$ and $|s|=n$ Since the edge $\mathrm{hm}$ does not occur in the path $e=\left(\begin{array}{ll}e_{1} e_{2} & e_{L}\end{array}\right)^{p} m$, neither $\mathrm{efghm}$ nor $\mathrm{ef} \mathrm{g} \mathrm{hm}$ can encroach upon itself or the other by any $n$ with $2 \leq n \leq p L+1$ If $p L+2 \leq n \leq$ $M-1$, and one of efghm or efg hm encroached upon the other by $n$, then the edge $m f$ would occur in the path $e$ for the following reason Since $|g h m|=|\bar{g} h m|=N+1$, the edge $m f$ would occur ending at position $n-(N+1)$ in the encroached-upon path But

$$
2 \leq(p L+2)-(N+1) \leq n-(N+1) \leq(M-1)-(N+1)=p L+1,
$$

which puts the edge $m f$ in the path $e$ Thus neither efghm nor efg $h m$ can encroach upon itself or the other by any $n$ with $2 \leq n \leq M-1$ Now efghm $\neq$ efg $h m$, so neither can encroach upon the other by $M$ Since $\mid$ efghm $\mid=M$, this proves the lemma

We define $\hat{\pi} \Sigma_{G} \rightarrow \Sigma_{H}$ as follows Let $x \in \Sigma_{G}$

If the block $t$ occurs in $x$, say ${ }_{t-M+1}(x)_{t}=t$, then

$$
{ }_{t-M+1}(\hat{\pi}(x))_{t}=\pi(\bar{t})=m \bar{v} m,
$$

If the block $\bar{t}$ occurs in $x$, say ${ }_{t-M+1}(x)_{\mathfrak{t}}=\bar{t}$, then

$$
1-M+1(\hat{\pi}(x))_{1}=\pi(t)=m v m,
$$

and for any coordinate $x_{l}$ of $x$ not occurring in a block $t$ or $\bar{t}$, set

$$
(\hat{\pi}(x))_{1}=\pi\left(x_{1}\right)
$$

By Lemma 41 , the strings $m v m$ and $m \bar{v} m$ in $H$ nontrivially overlap themselves or each other only at their end symbols Thus the strings $t$ and $\bar{t}$ can overlap each other or themselves in at most that many ways (in fact fewer ways), so $\hat{\pi}$ is well-defined as a $(2 M-1)$-block map $\hat{\pi} \quad \Sigma_{G} \rightarrow \Sigma_{H}$

Define functions $f$ and $\bar{f}$ with domain and range $\{1,2, \quad, d\}$ by

$$
f(l)= \begin{cases}\tau_{v}(l) & \text { if } l \neq 1, \\ 2 & \text { if } l=1,\end{cases}
$$

and

$$
\bar{f}(l)= \begin{cases}\tau_{\vec{v}}(t) & \text { if } l \neq 1, \\ 1 & \text { if } l=1\end{cases}
$$

Note that $\tau_{v}(1)=1$, so 1 is not in the range of $f$ Similarly, $\tau_{i}(1)=2$, so 2 is not in the range of $\bar{f}$ 
Let $m^{\prime} v^{\prime} m^{\tau_{v}(t)}, 1 \leq \imath \leq d$, be the paths in $G$ given by Lemma 32 that are $\pi$-labelled by $m v m$ Similarly, let $m^{\prime} \bar{v}^{\prime} m^{\tau_{\tilde{v}}(t)}$ be the paths in $G$ that are $\pi$-labelled by $m \bar{v} m$

We may denote

$$
m^{\prime} \hat{v}^{\prime} m^{f(t)}= \begin{cases}m v^{\prime} m^{\tau_{v}(t)} & \text { if } l \neq 1, \\ \bar{t}=m^{1} \bar{v}^{1} m^{2} & \text { if } l=1\end{cases}
$$

and

$$
m^{\prime} \hat{\bar{v}}^{\prime} m^{\bar{f}(t)}= \begin{cases}m^{t} \bar{v}^{t} m^{\tau_{\bar{v}}(t)} & \text { if } t \neq 1, \\ t=m^{1} v^{1} m^{1} & \text { if } \imath=1\end{cases}
$$

For a string $w$, denote ${ }_{0}[w]={ }_{0}[w]_{|w|-1}$

LEMMA 42

$$
\hat{\pi}^{-1}\left({ }_{0}[m v m]\right)=\bigcup_{t=1}^{d} 0\left[m^{\prime} \hat{v}^{t} m^{f(t)}\right]
$$

and

$$
\hat{\pi}^{-1}\left({ }_{0}[m \bar{v} m]\right)=\bigcup_{t=1}^{d}{ }_{0}\left[m^{i} \hat{\bar{v}}^{i} m^{\bar{f}(\mathrm{l})}\right]
$$

Proof The $2 d$ paths $m^{i} v^{t} m^{\tau_{v}(t)}$ and $m^{i} \bar{v}^{i} m^{\tau_{v}(t)}, 1 \leq \imath \leq d$ in the graph $G$ are $\pi$-labelled by $m v m$ or $m \bar{v} m$, so by Lemma 41 each of these paths non-trivially overlaps another or itself at most by one symbol (some $m^{t}$ ) In particular, each non-trivially overlaps $t$ and $\bar{t}$ by at most one symbol Thus, for $2 \leq \imath \leq d$,

$$
\hat{\pi}\left({ }_{0}\left[m^{\prime} v^{t} m^{\tau_{v}(t)}\right]\right) \subseteq \pi\left({ }_{0}\left[m^{\prime} v^{\prime} m^{\tau_{v}(t)}\right]\right)={ }_{0}[m v m],
$$

and

$$
\hat{\pi}\left({ }_{0}\left[m^{1} \bar{v}^{1} m^{2}\right]\right) \subseteq{ }_{0}[m v m]
$$

so

$$
\bigcup_{i=1}^{d} 0\left[m^{\prime} \hat{v}^{\prime} m^{f(1)}\right] \subseteq \hat{\pi}^{-1}(\mathrm{o}[m v m])
$$

On the other hand, if ${ }_{0}(\hat{\pi}(x))_{|m v m|-1}=m v m$ then either ${ }_{0}(x)_{|m v m|-1}=\bar{t}=m^{1} \bar{v}^{-1} m^{2}$ or ${ }_{0}(x)_{|m u m|-1}$ overlaps $t$ and $\bar{t}$ by at most one symbol, in which case $\hat{\pi}$ agrees with $\pi$ on ${ }_{0}(x)_{|m u m|-1}$, giving that ${ }_{0}(x)_{|m v m|-1}=m^{\prime} v^{\prime} m^{\tau_{2}(t)}$, where $2 \leq \imath \leq d$ This shows

$$
\hat{\pi}^{-1}\left({ }_{0}[m v m]\right) \subseteq \bigcup_{t=1}^{d}{ }_{0}\left[m^{\prime} \hat{v}^{\prime} m^{f(1)}\right]
$$

The barred version is proved similarly

Lemma 42 is the base case for an induction used to prove Lemma 43 below

Let $w$ be any path in the graph $H$ beginning and ending with the strings mum or $m \bar{v} m$ We can express

$$
w=m\left(w_{1} m\right)\left(w_{2} m\right) \quad\left(w_{k} m\right) \tilde{v} m,
$$


where

(1) $\tilde{v}=v$ or $\tilde{v}=\bar{v}$

(2) each $w_{j} m$ begins with $v m$ or $\bar{v} m$,

(3) the strings $m v m$ and $m \bar{v} m$ do not occur in any $w, m, 1 \leq \jmath \leq k$.

Note that $k=0$ if $w=m v m$ or $m \bar{v} m$ There is a unique decomposition satisfying (1), (2), and (3) because $m v m$ and $m \bar{v} m$ non-trivially overlap each other and themselves only in a single symbol $(m)$

LEMMA 43 Let $w$ be any path in $H$ beginning and ending with mum or mōm Let

$$
\boldsymbol{w}=\boldsymbol{m}\left(w_{1} m\right)\left(w_{2} m\right) \cdot\left(w_{k} m\right) \tilde{v} m
$$

be the decomposition defined above Then for $1 \leq 1 \leq d$,

$$
\begin{aligned}
& { }_{0}\left[m^{\prime}\right] \cap \hat{\pi}_{0}^{-1}[w]
\end{aligned}
$$

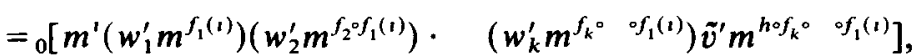

where $w_{\jmath}^{\prime}, 1 \leq \jmath \leq k$, and $\tilde{v}^{\prime}$ are paths in $G$ and

$$
f_{J}= \begin{cases}f & \text { if } w, m=v m \\ \bar{f} & \text { if } w, m=\bar{v} m \\ \tau_{u} \circ f & \text { if } w_{\jmath} m=v m u m \\ \tau_{u} \circ \bar{f} & \text { if } w_{\jmath} m=\bar{v} m u m\end{cases}
$$

and

$$
h= \begin{cases}f & \text { if } \tilde{v}=v, \\ \bar{f} & \text { if } \tilde{v}=\bar{v}\end{cases}
$$

Proof The proof is by induction on $k$. If $k=0$, then $w=m v m$ or $w=m \bar{v} m$ and this case follows from the equality

$$
\hat{\pi}^{-1}\left({ }_{0}[m v m]\right)=\bigcup_{i=1}^{d} 0\left[m^{\prime} \hat{v}^{\prime} m^{f(1)}\right]
$$

or

$$
\hat{\pi}^{-1}\left({ }_{0}[m \bar{v} m]\right)=\bigcup_{t=1}^{d}{ }_{0}\left[m^{\prime} \hat{\bar{v}}^{\prime} m^{\bar{f}(t)}\right]
$$

given by Lemma 42 Now suppose the lemma is true for all $0 \leq k<l$ and that

$$
w=m\left(w_{1} m\right)\left(w_{2} m\right) \quad\left(w_{1} m\right) \tilde{v} m
$$

Suppose that $w_{l} m$ begins with $v m$ (The argument for $\bar{v} m$ is similar) Set

$$
u=m\left(w_{1} m\right)\left(w_{2} m\right) \quad\left(w_{l-1} m\right) v m
$$

Then $u$ satisfies the inductive hypothesis, so

$$
{ }_{0}\left[m^{\prime}\right] \cap \hat{\pi}^{-1}[u]={ }_{0}\left[m^{\prime} \quad m^{g(t)} v^{\prime} m^{f \circ g(t)}\right],
$$

where $g=f_{l-1} \circ \quad \circ f_{1}$ There are two cases to consider

(1) $w_{l} m=v m$,

(2) $w_{l} m=v m u m$, where neither $m v m$ nor $m \bar{v} m$ occurs in mum

In case (1),

$$
\begin{aligned}
{ }_{0}\left[m^{\prime}\right] \cap \hat{\pi}^{-1}[w] & ={ }_{0}\left[m^{\prime}\right] \cap \hat{\pi}^{-1}[u] \cap \sigma^{-|u|+1} \hat{\pi}_{0}^{-1}[m \tilde{v} m] \\
& ={ }_{0}\left[m^{\prime} \quad m^{f \circ g(1)}\right]_{|u|-1} \cap|u|-1\left[m^{f \circ g(1)} \tilde{v}^{\prime} m^{h \circ f \circ g(1)}\right],
\end{aligned}
$$


so $f_{l}=f$ in this case In case (2),

$$
\begin{aligned}
{ }_{0}\left[m^{\prime}\right] & \cap \hat{\pi}_{0}^{-1}[w] \\
& ={ }_{0}\left[m^{\prime} \quad m^{g(t)} v^{\prime} m^{f \circ g(t)}\right]_{|u|-1} \cap \sigma^{-|u|+|m v m|} \hat{\pi}^{-1}{ }_{0}[m v m u m \tilde{v} m] \\
& ={ }_{0}\left[m^{\prime} \quad m^{g(t)} v^{\prime} m^{f \circ g(t)}\right]_{|u|-1} \cap{ }_{|u|-|m v m|}\left[m^{g(t)} v^{\prime} m^{f \circ g(t)} u^{\prime} m^{\tau_{u}{ }^{\circ}{ }^{\circ} g(t)} \tilde{v}^{\prime} m^{h \circ \tau_{u}{ }^{\circ} f \circ g(t)}\right],
\end{aligned}
$$

so $f_{l}=\tau_{u} \circ f$ in this case

Corollary 44 The map $\hat{\pi} \quad \Sigma_{G} \rightarrow \Sigma_{H}$ is onto

Proof By Lemma 43 , each path of the form (mvm) $u(m v m)$ in $H$ is the image by $\hat{\pi}$ of a path $\left(m^{\prime} v^{\prime} m^{f(1)}\right) u^{\prime}\left(m^{g(t)} v^{\prime \prime} m^{f \circ g(t)}\right)$ in $G$ Thus, by the irreducibility of $H$, any finite path in $H$ is the image by $\hat{\pi}$ of some path in $G$ It follows that the image of $\hat{\pi}$ in $\Sigma_{H}$ is dense, and by the compactness of $\Sigma_{G}$, that the image of $\hat{\pi}$ is all of $\Sigma_{H}$

Corollary 45 The map $\hat{\pi} \Sigma_{G} \rightarrow \Sigma_{H}$ is bounded-to-1

Proof As $\pi \Sigma_{G} \rightarrow \Sigma_{H}$ is bounded-to-1, $\Sigma_{G}$ and $\Sigma_{H}$ have the same entropy [CP] It follows from this, Corollary 44 , and [CP] that $\hat{\pi}$ is bounded-to- 1

CoROllary 46 If $\pi$ is right-closing, then so is $\hat{\pi}$

Proof Let $x, x^{\prime} \in \Sigma_{G}$ be left asymptotic points with $\hat{\pi}(x)=\hat{\pi}\left(x^{\prime}\right)$ We must show $x=x^{\prime}$ We may assume $x_{1}=x_{1}^{\prime}$ for $t \leq 0$ We may also assume (by replacing ${ }_{-x}(x)_{0}$ by some other past and shiftıng if necessary) that $-|m v m|+1(\hat{\pi}(x))_{0}=m v m$ If words from $\{m v m, m \bar{v} m\}$ occur infinitely often in $0(\hat{\pi}(x))_{\infty}$ then $x=x^{\prime} b$ an induction and Lemma 43 If words from $\{m v m, m \bar{v} m\}$ occur a finite number of times in ${ }_{0}(\hat{\pi}(x))_{\infty}$, let ${ }_{k-|m v m|+1}(\pi(x))_{k}$ be the final occurrence Then $x_{i}=x_{1}^{\prime}$ for $t \leq k$ by Lemma 43 Now ${ }_{k}(\hat{\pi}(x))_{\infty}={ }_{k}(\pi(x))_{\infty}$ by the definition of $\hat{\pi}$ off the blocks $t$ and $\bar{t}$ Similarly, ${ }_{k}\left(\hat{\pi}\left(x^{\prime}\right)\right)_{\infty}={ }_{k}\left(\pi\left(x^{\prime}\right)\right)_{\infty}$ So ${ }_{k}\left(\pi\left(x^{\prime}\right)\right)_{\infty}={ }_{k}(\pi(x))_{\infty}$, so $x=x^{\prime}$ because $\pi$ is right-closing

Corollary 47 The map $\hat{\pi} \Sigma_{G} \rightarrow \Sigma_{H}$ has lower degree than $\pi$ has

Proof Because the map $\hat{\pi}$ is not a 1-block map, we cannot apply verbatim the characterization of degree we gave in terms of the pre-image of a resolving block in $H$ However, we may choose an integer $q$ so that $\left|(m v)^{q} m\right|>2 M$ and observe that

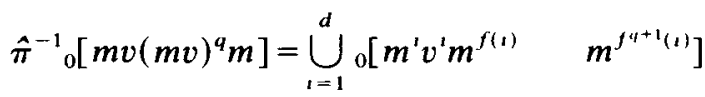

$$
\begin{aligned}
& \subseteq \bigcup_{t=1}^{d} \sigma_{0}^{-|m v|}\left[m^{f(t)} \quad m^{f^{4+1(t)}}\right],
\end{aligned}
$$

the last set being a disjoint union of at most $d-1\left|(m v)^{q} m\right|$-blocks Thus we may apply the criterion directly to the 1-block map

$$
\hat{\pi} \circ \psi_{2 M}^{-1} \Sigma_{G}^{[2 M]} \rightarrow \Sigma_{H},
$$

to conclude that the degree of $\hat{\pi}$ is at most $d-1$

From the assumption that the bounded-to-1 factor map $\pi \Sigma_{G} \rightarrow \Sigma_{H}$ has degree exceeding $p_{G} / p_{H}$, we have constructed a bounded-to-1 factor map $\hat{\pi} \Sigma_{G} \rightarrow \Sigma_{H}$ with 
degree less than the degree of $\pi$ Since the smallest possible degree of a factor map $\pi^{\prime}=\Sigma_{G} \rightarrow \Sigma_{H}$ is $p_{G} / p_{H}$, this shows that one could iterate the construction to get a factor map degree exactly $p_{G} / p_{H}$, proving Theorem 11

\section{The sofic case}

A sofic system is a symbolic system that is a factor of a shift of finite type In fact any sofic system is a factor by a 1-to-1 almost everywhere map of a shift of finite type [F]

Theorem 11 can be generalized to the case of sofic domain and range

THEOREM 51 If $\pi S \rightarrow T$ is a bounded-to-1 factor map from an irreducible sofic system $S$ with period $p_{S}$ to an trreductble sofic system $T$ with period $p_{T}$, then there is a factor map $\hat{\pi} S \rightarrow T$ that is $\left(p_{S} / p_{T}\right)$-to-1 almost everywhere Moreover, if $\pi$ is right closing, then $\hat{\pi}$ may be taken to be right closing also

Here, the period of a sofic system is the period of any 1-to-1 almost everywhere finite type extension

The proof of Theorem 51 is largely the same as the proof of Theorem 11 The only real change is that we replace resolving blocks by their appropriate generalization in the sofic setting markov magic words [B]

We follow [B] in the following two definitions

Given a sofic system $T$, a markov word for $T$ is an allowable word $w$ such that if $u w$ and $w v$ are allowable words in $T$, then so is $u w v$

Given a bounded-to-1 1-block factor map $\pi S \rightarrow T$ from an irreducible sofic system $S$ to an irreducible sofic system $T$, define $\mathscr{W}$ to be the set of allowable words $w$ in $T$ for which

(1) $w$ is a markov word for $T$,

(11) $\pi_{0}^{-1}[w] \subseteq \bigcup_{t=1}^{d}\left[w^{t}\right]_{k}$, where $k \geq_{J}$ and $w^{1}, w^{2}, \quad, w^{d}$ are markov words for $S$ In [B] it is shown that $\mathscr{W}$ is non-empty Any $w \in \mathscr{W}$ for which $d$ in (11) is minimal is called a markov magic word for $\pi S \rightarrow T$ The minimal $d$ is the degree of the factor map $\pi \quad S \rightarrow T$ [B]

We may use [B, Proposition 14] and a construction sımilar to that of $\S 3$ above (from [KMT]) to reduce to the case where $\pi S \rightarrow T$ has a markov magic symbol $m$ Then [B, Proposition 14 ] gives the following generalization of the permutation Lemma 22

LEMMA 52 Let $\pi S \rightarrow T$ be a degree d 1-block map with markov magic symbol $m$ Let $m^{1}, m^{2}, \quad, m^{d}$ be the symbols in $S$ with $\pi^{-1}{ }_{0}[m]=\bigcup_{i=1}^{d}\left[m^{\prime}\right]$ For each allowable word of the form mum in $T$, there are $d$ words $u^{1}, u^{2}, \quad, u^{d}$ in $S$ and a permutation $\tau_{u}$ of $\{1,2, \quad, d\}$ such that

$$
\pi^{-1}{ }_{0}[m u m]=\bigcup_{1=1}^{d}{ }_{0}\left[m^{\prime} u^{\prime} m^{\tau_{u}^{(1)}}\right]
$$

As in the shift of finite type case, we may assume that the symbol $m$ in $T$ has at least two predecessors and two successors 
The period of $T$ is

$$
\operatorname{gcd}\{|\operatorname{mum}|-1 \text { mum is a word in } T\}
$$

and the period of $S$ is

$$
\operatorname{gcd}\left\{\left|m^{1} u m^{1}\right|-1 m^{1} u m^{1} \text { is a word in } S\right\}
$$

The construction of $\hat{\pi} S \rightarrow T$ using Lemma 52 follows much the same lines as the shift of finite type case

\section{The Markov chain case}

If the irreducible shift of finite type $\left(\Sigma_{G}, \sigma\right)$ is given a Markov measure $\mu_{G}$ defined by a stochastic matrix $P \geqslant 0$ via

$$
\mu_{G}\left({ }_{0}[s t]\right)=P_{s t} \mu_{G}\left({ }_{0}[s]\right),
$$

then $\left(\Sigma_{G}, \sigma, \mu_{G}\right)$ is called a Markov chain

Following [PS], define the weight of a cycle $s_{0} s_{1} \quad s_{p-1}$ in the graph $G$ as

$$
w_{G}\left(s_{0} s_{1} \quad s_{p-1}\right)=P_{s_{0} s_{1}} P_{s_{1} s_{2}} \quad P_{s_{p-1} s_{0}},
$$

and the multiplicative subgroup $\Delta_{G}$ of $\mathbb{R}^{+}$by

$$
\Delta_{G}=\left\{\frac{W_{G}(s)}{W_{G}\left(s^{\prime}\right)} s, s^{\prime} \text { are cycles in } G \text { with }|s|=\left|s^{\prime}\right|\right\}
$$

In [PS] it is shown that if

$$
\pi\left(\Sigma_{G}, \sigma, \mu_{G}\right) \rightarrow\left(\Sigma_{H}, \sigma, \mu_{H}\right)
$$

is measure-preserving, then $\Delta_{G} \subseteq \Delta_{H}$, moreover, if $\pi$ is 1-to-1 almost everywhere, then $\Delta_{G}=\Delta_{H}$

As was pointed out to me by Brian Marcus, the construction of $\hat{\pi}$ used in the proof of Theorem 11 can be adapted to work in the category of Markov measurepreserving block maps to give a partial converse to the [PS] result

THEOREM 61 If $\pi\left(\Sigma_{G}, \sigma, \mu_{G}\right) \rightarrow\left(\Sigma_{H}, \sigma, \mu_{H}\right)$ is a measure-preserving factor map from the Markov chain $\Sigma_{G}$ with period $p_{G}$ to a Markov chain $\Sigma_{H}$ with equal period $p_{H}=p_{G}$, and if $\Delta_{G}=\Delta_{H}$, then there is a measure-preserving factor map $\hat{\pi}\left(\Sigma_{G}, \sigma, \mu_{G}\right) \rightarrow\left(\Sigma_{H}, \sigma, \mu_{H}\right)$ that is 1-to-1 almost everywhere

Sketch of proof In the proof of Theorem 11 , we construct paths $t$ and $\bar{t}$ in the graph $G$ such that their images $\pi(t)=m v m$ and $\pi(\bar{t})=m \bar{v} m$ in the graph $H$ overlap by at most one symbol The map $\hat{\pi} \Sigma_{G} \rightarrow \Sigma_{H}$ is defined by "switching the images" of $t$ and $\bar{t}$

Now $v m$ and $\bar{v} m$ are both cycles in the graph $H$ If $w_{H}(v m)=w_{H}(\bar{v} m)$ then $\hat{\pi}$, like $\pi$, will be measure-preserving Otherwise the ratio

$$
\frac{w_{H}(v m)}{w_{H}(\bar{v} m)}=\rho \in \Delta_{H}=\Delta_{G}
$$

is equal to a ratio

$$
\frac{w_{G}(\bar{r})}{w_{G}(r)}=\rho \in \Delta_{G},
$$


where $r=s_{0} r_{1} r_{2} \quad r_{k}$ and $\bar{r}=s_{0} \bar{r}_{1} \bar{r}_{2} \quad \bar{r}_{k}$ are cycles in the graph $G$ based at the state $s_{0}$ of $G$ defined in the proof of Theorem 11

Now interpolate the cycle $\bar{r}$ into the path $\bar{t}$ at state $s_{0}$, and interpolate the cycle $r$ into the path $t$ at state $s_{0}$, and extend the common prefix $c_{1} c_{2} \quad c_{p L+1}$ of $t$ and $\bar{t}$ (by choosing a larger $L$ if necessary) to ensure that the two modified paths $t^{\prime}$ and $\vec{t}$, like $t$ and $\vec{t}$, non-trivially overlap themselves or each other only by one symbol Denote $\pi\left(t^{\prime}\right)=m v^{\prime} m$ and $\pi\left(\bar{t}^{\prime}\right)=m \bar{v}^{\prime} m$

Now

$$
w_{H}(\pi(r))=w_{G}(r)
$$

and

$$
w_{H}(\pi(\bar{r}))=w_{G}(\bar{r}),
$$

so

$$
\frac{w_{H}\left(v^{\prime} m\right)}{w_{H}\left(\bar{v}^{\prime} m\right)}=\frac{w_{H}(v m) w_{G}(r)}{w_{H}(\bar{v} m) w_{G}(\bar{r})}=1,
$$

by the choice of the cycles $r$ and $\bar{r}$ Hence if we define $\hat{\pi} \Sigma_{G} \rightarrow \Sigma_{H}$ by 'switching the images' of $t^{\prime}$ and $\bar{t}^{\prime}$ (which we can do since $t^{\prime}$ and $\bar{t}^{\prime}$ non-trivially overlap each other or themselves by at most one symbol), then $\hat{\pi}$, like $\pi$, will be measure-preserving As in the proof of Theorem 11, $\hat{\pi}$ will have lower degree than $\pi$

Acknowledgements The author is indebted to Roy Adler, Brian Marcus, and Bruce Kitchens for posing the problem and for useful discussions

\section{REFERENCES}

[AGW] R Adler, L W Goodwyn \& B Weiss Equivalence of topological Markov Shifts Israel $J$ Math 27 (1977) 49-63

[AM] R Adler \& B Marcus Topological entropy and equivalence of dynamical systems Memoirs Amer Math Soc 219 (1979)

[B] $\quad M$ Boyle Constraints on the degree of a sofic homomorphism and the induced multiplication of meaures on unstable sets Israel $J$ of Math 53 (1986) 52-68

[BKM] M Boyle, B Kitchens \& B Marcus A note on mınımal covers for sofic systems Proc Amer Math Soc 95 (1985) 403-411

[BMT] M Boyle, B Marcus \& P Trow Resolving maps and the dimension group for shifts of finite type Memoirs AMS 377 (1987)

[CP] E Coven \& M Paul Endomorphisms of irreducible shifts of finite type Math Svstems Theory 8 (1974) $167-175$

[F] R Fischer Sofic systems and graphs Monatch fur Math 80 (1975) 179-186

[KMT] B Kitchens, B Marcus \& P Trow Eventual factor maps and compositions of closing maps, in preparation

[PS] W Parry \& K Schmidt Natural coefficients and invariants for Markov shifts Invent Math 76 (1984) $15-32$ 